\title{
Linee guida e SIPMeL
}

\section{Clinical Practice Guidelines and SIPMeL (Italian Society of Clinical Pathology and Laboratory Medicine)}

\author{
Piero Cappelletti ${ }^{1}$
}

Ricevuto: 10 dicembre 2015 / Accettato: 17 dicembre 2015

(c) Springer-Verlag Italia 2015

Riassunto Dalla prima definizione di linee guida (LG), da parte dell'Institute of Medicine (IOM) venticinque anni fa, una molteplicità di Istituzioni pubbliche e private si è occupata di definire raccomandazioni, caratteristiche e attributi, metodi di costruzione e di validazione, modi di espressione della forza delle raccomandazioni e del livello delle evidenze. Tuttavia lo IOM, con il suo documento Clinical Practice Guidelines We Can Trust (2011), resta il riferimento più sicuro. In Medicina di Laboratorio, la definizione di LG è resa particolare dalla scarsità di "evidenze" basate su "outcome" correlabili e dalla necessità di affrontare problemi "interni" alla produzione delle informazioni cliniche. Anche qui vi è una molteplicità di attori, tra cui emerge come riferimento la National Academy of Clinical Biochemistry (NACB) con le sue Laboratory Medicine Practice Guidelines (LMPG), che nel 2014 ha rilasciato un documento che può essere considerato le "linee guida delle linee guida". In questo quadro articolato e complesso, il ruolo di una Società scientifica italiana, attraverso i suoi Gruppi di Studio, può essere quello di misurarsi con la definizione di LG nazionali e internazionali, quando ne ha la forza, di collaborare con le Società scientifiche disciplinari per più ampi "consensus", di revisionare in chiave italiana LG internazionali e di adattarle alla realtà del Laboratorio, di definire raccomandazioni per quei passaggi del total testing process trascurati dalle LG generali sia nella fase preanalitica, sia in quella analitica, sia in quella postanalitica per rispondere al meglio al quesito clinico.

Parole chiave Linee guida $\cdot$ IOM $\cdot$ LMPG $\cdot$ AGREE . GRADE

\footnotetext{
P. Cappelletti pie.cappelletti@gmail.com

1 SIPMeL, Castelfranco Veneto, TV, Italia
}

\begin{abstract}
Summary Since the first definition of Clinical Practice Guidelines (CPG) by Institute of Medicine (IOM) in 1990, several public and private institutions have proposed definitions of recommendations, attributes and characteristics of CPG, methods for building, evaluating and validating evidences and CPG, tools for grading and weighting practice recommendations. Nevertheless, the IOM document Clinical Practice Guidelines We Can Trust (2011) is still the reference work. In Laboratory Medicine, the definition of guidelines is influenced by paucity of evidences of outcomes clearly related to laboratory tests and by necessity of guidance for "internal" issues in the production of the laboratory information to the clinic. In that field, National Academy of Clinical Biochemistry (NACB) with its Laboratory Medicine Practice Guidelines (LMPG) and its document Standard Operating Procedures (2014) has a central role. In this complex framework, an Italian Scientific Society and its Working Groups may play a significant role in defining national or even international CPG, collaborating with clinical Scientific Societies for consensus, reviewing international CPG for targeting the right audience and the right means of implementation in Italian environment, preparing specific recommendations for the steps of total testing process, ignored by general CPG, to answer adequately to the clinical question.
\end{abstract}

Keywords Guidelines $\cdot I O M \cdot L M P G \cdot A G R E E \cdot G R A D E$

\section{Introduzione}

Le linee guida (LG) sono, per definizione [1], strumenti di aiuto sistematico per assumere decisioni mediche complesse, che, quando sviluppate rigorosamente con un processo 
trasparente che combina l'evidenza scientifica con l'esperienza clinica e i valori dei pazienti, hanno la potenzialità di migliorare la qualità dell'assistenza e gli esiti della cura. Le LG sono strettamente correlate allo sviluppo dell'Evidencebased Medicine (EBM) e ai suoi strumenti, in particolare le revisioni sistematiche (SR), anche se si ripromettono di offrire indicazioni valide anche nelle aree dalle evidenze scarse, deboli o addirittura assenti, attraverso ampie e articolate procedure di consenso. In Medicina di Laboratorio i diversi livelli di raccomandazioni si prefiggono il miglioramento dell'esito medico (outcome) per il paziente attraverso la validità, sicurezza, appropriatezza e convenienza dell'informazione medica, trasferita ai curanti e ai pazienti, e dei mezzi di sua produzione.

Molteplici sono le organizzazioni (Istituzioni pubbliche e private, Società scientifiche) che producono LG. Possiamo citare, tra le Istituzioni, in ambito americano il Guidelines International Network (G-I-N) [2] di International Guidelines Library, che è la raccolta di LG più grande al mondo, tenuta da un'organizzazione privata non-profit, e la National Guidelines Clearinghouse [3] della Agency for Healthcare Research and Quality (AHRQ) USA e in ambito anglosassone la raccolta di Published Clinical Guidelines del National Institute of Health and Clinical Excellence (NICE) [4] del NHS UK, lo Scottish Intercollegiate Guidelines Network (SIGN) [5] del NHSQI Scotland e il New Zeland Guidelines Group (NZGG) [6] del Ministero della salute neozelandese. E tra le Società scientifiche le più attrezzate sono quelle cardiologiche americane [7] ed europee [8], quelle americane toraciche [9] e oncologiche [10]. Tuttavia, il più sicuro riferimento è l'attività in questo campo dell'Institute of Medicine (IOM) [11].

In Italia, il Decreto legislativo 229/99, che vincola "l'erogazione dell'assistenza, dei servizi e delle prestazioni sanitarie da parte del SSN a prove scientifiche di significativo beneficio in termini di salute" e che affida "Al Piano Sanitario Nazionale ... il compito di indicare un primo gruppo di linee guida e i relativi percorsi terapeutici", e il Piano Sanitario Nazionale 2003-2005, che definisce i servizi essenziali come "accettabili sul piano sociale nonché tecnicamente appropriati ed efficaci, in quanto fondati sulle prove di evidenza ed erogati nei modi economicamente più efficienti", hanno determinato il contesto normativo in cui ha operato il Programma Nazionale per le Linee Guida (PNLG) avviato nel 1998 con il coordinamento dell'Agenzia per i Servizi sanitari regionali e l'Istituto superiore di sanità (ISS). Nel 2006 è stato siglato un accordo tra la Direzione generale della programmazione sanitaria del Ministero della salute e l'ISS per un programma dal titolo "Sistema Nazionale Linee Guida: produzione di linee guida e strategie di implementazione" (SNLG-ISS) [12]. Il centro operativo del programma è istituito presso il Centro Nazionale di Epidemiologia, Sorveglianza e Promozione della Salute dell'ISS, specificatamen- te presso il Reparto di Epidemiologia Clinica e Linee Guida e risponde a un comitato strategico del Ministero della salute. Collaborano al programma l'ASL 6 di Livorno, l'Agenzia di Sanità Pubblica della regione Lazio, il Centro per la Valutazione dell'Efficacia dell'Assistenza Sanitaria della USL di Modena, il Centro Cochrane Italiano.

\section{Le linee guida per la pratica clinica}

Nel 1990 lo IOM [13] ha definito le Linee Guida per la Pratica Clinica (Clinical Practice Guidelines, CPG) come "raccomandazioni sviluppate in modo sistematico per assistere medici e pazienti nelle decisioni sulla gestione appropriata di specifiche condizioni cliniche" (systematically developed statements to assist practitioner and patient decisions about appropriate health care for specific clinical circumstances) e nel suo documento Guidelines for Clinical Practice: From Development to Use [14], pubblicato nel 1992, ha adottato le otto caratteristiche che le CPG devono possedere: validità, riproducibilità, applicabilità clinica, flessibilità clinica, chiarezza, multidisciplinarietà, revisione programmata $\mathrm{e}$ documentazione.

Le CPG, secondo la visione dello IOM negli anni Novanta, sono basate sulla dichiarazione della forza delle evidenze scientifiche e del giudizio degli esperti che le sostengono, forniscono una spiegazione del razionale di ogni deviazione dall'evidenza scientifica, sono prodotte da un processo che ingloba varie forme di partecipazione di tutti gli stakeholders e tengono in considerazione i problemi dell'implementazione e della loro valutazione dall'inizio del processo di formazione.

Negli anni successivi il numero delle LG e dei gruppi produttori di CPG è andato aumentando enormemente, al punto che, alla fine del primo decennio degli anni Duemila, il database $G-I-N$ ne comprendeva circa 4000. E, d'altra parte, lo stato dello sviluppo di CPG non ha pienamente mantenuto le promesse iniziali. Infatti, l'eccessiva molteplicità dei produttori è risultata talora in uno spreco di risorse, spesso in una variabilità di metodi di qualità incerta e quasi sempre in un mancato coordinamento di argomenti e indirizzi. La maggior parte delle LG non pare rispondere alle differenti necessità dei diversi stakeholders, soprattutto relativamente a garanzia di qualità, controllo dei costi, confronto con pratiche alternative e riduzione della responsabilità medica. Il valore educativo delle LG, inoltre, è limitato spesso dalla mancanza di esplicite dimostrazioni di evidenze e di "razionale" [15]. L'efficacia clinica delle LG è largamente indeterminata, data la limitatezza degli studi sul loro impatto clinico [16]. Molte, infine, sono le barriere che ostacolano la loro implementazione e penetrazione [17]. E, più recentemente, è emerso in tutta la sua gravità il problema del conflitto di interessi, affrontato dallo IOM nei report Knowing What 
Works in Health Care (IOM, 2008) [16] e Conflict of Interest in Medical Research, Education, and Practice (IOM, 2009) [18].

Di conseguenza, lo IOM, in collaborazione con AHRQ, su mandato del Congresso USA (Medicare Improvements for Patients and Providers Act, 2008), ha pubblicato nel documento Clinical Practice Guidelines We Can Trust [19] del 2011 otto standard che rispondono ad alcuni essenziali problemi delle CPG, quali la trasparenza, il conflitto di interessi, la composizione del gruppo, l'intersezione tra CPG e SR, la definizione dei fondamenti dell'evidenza e della forza delle raccomandazioni, l'articolazione delle raccomandazioni, le revisioni esterne e la manutenzione nel tempo.

In questo documento, le LG per la pratica clinica sono definite come "documenti che includono raccomandazioni tese a ottimizzare la cura dei pazienti e che sono basate sulla revisione sistematica delle evidenze e una valutazione dei benefici e danni delle alternative di cura" (clinical practice guidelines are statements that include recommendations intended to optimize patient care that are informed by a systematic review of evidence and an assessment of the benefits and harms of alternative care options). La nuova definizione pone una chiara distinzione tra CPG e altre forme di guida clinica derivate da processi largamente diversi, quali le dichiarazioni di consenso, le indicazioni di esperti e i criteri di appropriatezza, che pure possono avere una loro validità in particolare in quelle aree cliniche in cui prove di efficacia di alta qualità sono limitate o del tutto assenti.

Secondo lo IOM, per essere valide, le LG devono: basarsi sulla revisione sistematica delle prove di efficacia disponibili; essere sviluppate da un autorevole gruppo multidisciplinare di esperti e rappresentanti dei principali gruppi interessati; prendere in considerazione i sottogruppi rilevanti di pazienti e le preferenze dei pazienti, se necessario; basarsi su un processo esplicito e trasparente che riduca al minimo le distorsioni, i bias e i conflitti di interesse (a questo è dedicata un'ampia sezione degli standard); fornire un'accurata illustrazione delle relazioni logiche tra opzioni assistenziali alternative ed esiti di salute e fornire una classificazione sia della qualità delle prove sia della forza delle raccomandazioni; essere riconsiderate e aggiornate, se necessario, quando nuove rilevanti prove di efficacia giustifichino modifiche delle raccomandazioni.

\section{Linee guida e Medicina di Laboratorio}

La definizione di LG in Medicina di Laboratorio presenta specifici problemi. Principalmente, da un lato, la difficoltà di correlare l'utilizzo di esami di laboratorio agli esiti clinici del paziente e quindi di avere studi primari di alta qualità, come sono per definizione i trials clinici randomizzati (RCT), sposta l'attenzione verso esiti surrogati co- me l'accuratezza diagnostica, peraltro non sempre facilmente perseguibili [20]. Dall'altro, nella pratica del laboratorio sono spesso gli aspetti tecnologici e organizzativi che hanno la necessità di essere indirizzati (guidance), soprattutto quando questi aspetti che possono apparire "interni" rispetto all'interfaccia clinica-laboratorio condizionano invece pesantemente il percorso diagnostico e quindi terapeutico del paziente [21].

Anche in Medicina di Laboratorio sono molteplici gli attori che si dedicano all'indicazione di raccomandazioni sia sulla sponda americana dell'Atlantico (College of American Pathologists) [22] che in quella inglese (Royal College of Pathologists [23] e Association of Clinical Biochemistry [24]) o alla definizione e raccolta di LG di Laboratori in ambiti specifici, come International Society for Laboratory Hematology (ISLH) [25] per l'ematologia o la European Society of Clinical Microbiology and Infectious Diseases (ESCMID) [26] per la microbiologia. Tuttavia, il riferimento più generale e articolato è Laboratory Medicine Practice Guidelines (LMPG) di National Academy of Clinical Biochemistry (NACB) [27]. Dal 2010, un'organizzazione ad hoc in collaborazione con American Association of Clinical Chemistry (AACC) - il nuovo AACC/NACB Evidence-Based Laboratory Medicine Committee (EBLMC)—ha iniziato a rivedere procedure operative standard e raccomandazioni pratiche alla luce dei documenti IOM del 2011 [19, 28]. Nel 2014, infine, NACB ha pubblicato un documento [29] che può essere considerato la "linea guida delle linee guida" per la medicina di laboratorio: Standard Operating Procedures for Preparing, Publishing and Revising National Academy of Clinical Biochemistry Laboratory Medicine Practice Guidelines. Including Review and Approval of External Society/Organization Guidelines for Endorsement and Support by $A A C C / N A C B$.

Le LMPG sono raccomandazioni pratiche documentate, sviluppate con un approccio evidence-based, utilizzando quanto più possibile $\mathrm{SR}$, e indirizzate a risolvere questioni riguardanti l'appropriato uso degli esami di laboratorio in una specifica disciplina scientifica e/o clinica al fine di ottimizzare la cura dei pazienti.

Sono prodotte da un comitato di esperti di laboratorio e clinici con un coordinatore, scelti da EBLMC, su temi selezionati da NACB, spesso in collaborazione con altri soggetti, tenendo conto di un'ampiezza ragionevole e dell'emergenza del tema stesso. Quando fattibile, esse tengono conto dei benefici e danni di approcci laboratoristici alternativi e delle indicazioni 2011 dello IOM, sapendo che non sempre sono tutte applicabili per ogni singola raccomandazione.

Innanzitutto, poiché una ricerca [30] del 2012 ha mostrato la diseguale qualità delle LMPG, il primo passaggio è la valutazione delle LG correlate già esistenti utilizzando $A p$ praisal of Guidelines for Research \& Evaluation (AGREE e AGREE II) [31], una check-list di 23 items raggruppati in 6 
aree principali (campo di applicazione e finalità; coinvolgimento degli stakeholders; rigore dello sviluppo; chiarezza e presentazione; applicabilità; indipendenza editoriale), nella revisione del 2010 (AGREE II) e con lo strumento disponibile on line [32] e quindi eseguendo o commissionando una SR su database elettronici (come PubMed, Index Medicus, OVID database, e-mail alert), letteratura grigia (come poster, atti, presentazioni power-point) e collezioni di EBM (come Cochrane Collaboration o National Guidelines Clearinghouse).

In secondo luogo, per la produzione di raccomandazioni un tema centrale è l'espressione della forza delle stesse e del livello di evidenza. Il metodo oggi più in voga è il Grading of Recommendations, Assessment, Development and Evaluation (GRADE) [33] presentato in una serie di lavori su BMJ dall'aprile del 2008 in poi e accettato anche dalla World Health Organization (WHO). Nonostante un articolo specificamente dedicato alla diagnostica [34], GRADE non è stato considerato adatto per le raccomandazioni di laboratorio e quindi NACB adotta le indicazioni di $U S$ Preventive Services Task Force [35] modificate.

La Forza delle Raccomandazioni si articola in:

- A: NACB raccomanda fortemente l'adozione in quanto vi è una buona evidenza (prove di efficacia) che così si migliorino gli esiti e i benefici superino decisamente $i$ rischi;

- B: NACB raccomanda l'adozione in quanto c'è almeno una discreta evidenza che così si migliorino gli esiti e i benefici superino i rischi;

- C: NACB non raccomanda l'adozione perché ci sono prove di inefficacia o di rischi maggiori dei benefici;

- I: NACB conclude che le evidenze sono insufficienti, di qualità scarsa o confliggenti e il rapporto benefici/rischi non è stabilito così che non possono essere definite raccomandazioni fondate.

Il Livello delle Evidenze è così graduato:

- I: evidenze fondate su studi ben disegnati e ben condotti in popolazioni rappresentative;

- II: evidenze sufficienti ma limitate in numero, qualità, consistenza degli studi individuali; generalizzabili nella pratica; o di natura indiretta;

- III: evidenze insufficienti per determinare effetti sugli esiti clinici per la limitatezza in numero o forza degli studi, debolezza di disegno o svolgimento, buchi nella catena delle evidenze o mancanza di informazioni.

Infine, le raccomandazioni vengono formalmente decise dal gruppo, confrontate con altre organizzazioni se del caso, messe in discussione pubblica on line, presentate in un incontro ad hoc o alla Arnold O. Beckman Conference, incontro annuale di AACC, e pubblicate su Clinical Chemistry e/o su altri giornali peer-reviewed, con la dichiarata tempistica delle revisioni e delle verifiche di applicazione.
Linee guida di Medicina di Laboratorio e SIPMeL

In questo quadro articolato e complesso, ricco di iniziative di grande respiro internazionale, c'è posto per un'attività di LG e raccomandazioni di pratica clinica in Medicina di Laboratorio da parte di una Società Scientifica italiana?

Come si è cercato di descrivere, le LG internazionali e, nello specifico, le LMPG sono scritte con massima attenzione alle fasi della raccolta delle evidenze, della gradazione della forza, del coinvolgimento degli stakeholders e della loro adozione, diffusione, disseminazione e implementazione e, infine, della verifica. Però il tema dell'effettivo utilizzo delle LG da parte dei professionisti resta del tutto aperto [36], nonostante le molteplici procedure utilizzate [37], e l'inadeguata definizione del target della disseminazione e delle strategie educazionali è la principale ragione del fallimento dell'implementazione di tante LG [24].

La SIPMeL, come recita l'art. 5 dello Statuto [38], "intende rappresentare, per la Medicina di Laboratorio, un Forum multidisciplinare e multiprofessionale di idee e di esperienze per il progresso delle scienze di laboratorio e per una loro applicazione pratica e, per la società civile, uno stabile riferimento per la conoscenza, lo sviluppo e l'organizzazione dell' attività della Medicina di Laboratorio, al servizio dei cittadini”. E in relazione a ciò, intende perseguire, tra l'altro, "l'elaborazione di linee guida, anche in collaborazione con ASSR (Agenzia per i Servizi Sanitari Regionali) e FISM (Federazione delle Società Medico-Scientifiche Italiane) e altre entità scientifiche riconosciute" (comma 5.3) e "la promozione di studi e di ricerche scientifiche finalizzate e di sperimentazione gestionale" (comma 5.4).

A questi obiettivi si dedicano specificatamente i Gruppi di Studio (GdS), il cui Regolamento [39] ricorda, all'art. 10, che: "L'attività dei GdS deve riguardare tutta la complessa realtà del laboratorio e va orientata verso la definizione di linee guida per gli aspetti della buona pratica di laboratorio e per l'utilizzo clinico degli esami di laboratorio: tale attività, fondamentale per la mission della Società, va focalizzata in aree di interesse sub disciplinare. Il GdS collabora con la Commissione per la Formazione Continua per far conoscere le linee guida al maggior numero possibile di professionisti italiani e per promuoverne l'applicazione nelle diverse realtà". La Società assume la piena rappresentazione delle LG, come indica l'art. 14 del citato Regolamento: "I risultati dell' attività dei GdS sono patrimonio comune della Società. I risultati vengono comunicati a tutti i Soci e all'intera Comunità scientifica, tramite la loro pubblicazione sulla Rivista, in occasione di manifestazioni scientifiche pubbliche (convegni, simposi, tavole rotonde ecc.) della SIPMeL e in uno specifico spazio all'interno del Congresso Nazionale. Nel caso di risultati che rappresentano ufficialmente il parere della Società (raccomandazioni, LG ecc.), 
dopo una loro valutazione da parte del Coordinatore Generale dei GdS, essi dovranno essere discussi e approvati dal Consiglio Nazionale".

Una ricerca bibliografica nella nostra Rivista degli ultimi 15 anni restituisce circa 150 articoli che presentano o che trattano LG. La produzione di LG è attività che richiede molto tempo, competenze specifiche (tecnologiche, metodologiche e cliniche), gruppi multidisciplinari e assenza di conflitto di interessi. Alcuni GdS sono sufficientemente "forti" da misurarsi con la definizione di LG destinate a indirizzare i professionisti nazionali o internazionali rispetto a specifiche questioni; altri collaborano attivamente con le Società scientifiche cliniche di disciplina o di area per la definizione di raccomandazioni nazionali. L'applicazione alla realtà italiana di revisioni di LG internazionali è dettata dalla peculiarità della situazione italiana e dalla necessità di tagliare su misura del target le LG, ma è anche spesso necessaria alla luce dei tempi di obsolescenza delle LG conosciuti (mediana di obsolescenza 5,5 anni, ma primi segni per almeno un quarto di esse dopo 2 anni) [40]. Questo lavoro di adattamento locale e d'implementazione è ampiamente consigliato dagli organismi e dai documenti internazionali, anche perché molte LG di buona qualità non vengono implementate e perché le barriere a diffusione e implementazione possono risiedere nei destinatari sotto forma di disinteresse o resistenze ma spesso nelle debolezze dei metodi di knowledge translation e nel ruolo dei messaggeri (opinion maker) $[17,36]$.

D'altra parte, tenuto conto dell'enorme quantità di LG prodotte e del livello dei produttori internazionali, molto spesso sarebbe arrogante pensare di preparare LG per la pratica clinica, mentre è più saggio utilizzare, in maniera sistematica e ragionata, quanto disponibile per fornire raccomandazioni utili ai professionisti della Medicina di Laboratorio a risolvere concreti problemi giornalieri che consentono risultati e risposte efficaci nel percorso clinico del paziente. Infatti, la maggior parte delle attività di raccomandazione si focalizza principalmente ad aspetti di guidance in tutte le fasi del total testing process e in particolare di quelle fasi e di quei passaggi trascurati dalla LG cliniche che si focalizzano prevalentemente sull'appropriatezza della richiesta di test diagnostici e del loro ruolo nel percorso diagnostico, di rule in/rule out e di follow-up (fase preanalitica diversa dalla richiesta dell'esame; fase analitica; fase postanalitica interna al Laboratorio) [21]. Infatti, è noto [21] come le LG cliniche abbiano generalmente una bassa considerazione per gli aspetti considerati laboratoristici, rispettando in media solo in un terzo dei casi le necessarie informazioni riguardo lo stato dei pazienti, le interferenze biologiche e analitiche e il trattamento dei campioni, e che l'inserimento di uno specialista di laboratorio nei comitati/gruppi di preparazione di LG cliniche migliora la situazione rendendone circa la metà esaustive sotto il profilo dei mezzi diagnostici. È quindi spesso necessario "adattare" LG cliniche alle necessità del Laboratorio.
Poiché il lavoro all'interfaccia clinica-laboratorio è considerato centrale nella visione attuale della Medicina di Laboratorio [41], l'impegno della Società e dei suoi GdS è nella direzione di rispondere adeguatamente al suo ruolo all'interno del percorso complesso e cumulativo rappresentato dalla Medicina Traslazionale e deve tradursi in indicazioni, suggerimenti e raccomandazioni che rendano più veloce l'implementazione di approcci up-to-date ed evidencebased del singolo professionista o, meglio, dell'equipe di laboratorio a tutte le fasi del total testing process, per rispondere adeguatamente al quesito clinico esplicito o implicito in ogni richiesta di esame di laboratorio [42].

\section{Conflitti di interesse Nessuno.}

Studi condotti su esseri umani e animali L'articolo non contiene alcuno studio eseguito su esseri umani e su animali da parte dell' autore.

\section{Bibliografia}

1. Oosterhuis WP, Bruns DE, Watine J et al (2004) Evidence-based guidelines in laboratory medicine: principles and methods. Clin Chem 50:806-818

2. http://www.g-i-n.net/library/international-guidelines-library (Accesso 1 dicembre 2015)

3. https://www.guideline.gov (Accesso 1 dicembre 2015)

4. https://www.nice.org.uk/guidance (Accesso 1 dicembre 2015)

5. http://www.sign.ac.uk (Accesso 1 dicembre 2015)

6. https://www.health.govt.nz (Accesso 1 dicembre 2015)

7. https://www.acc.org/guidelines (Accesso 1 dicembre 2015)

8. https://www.escardio.org (Accesso 1 dicembre 2015)

9. https://www.chestnet.org/Guidelines (Accesso 1 dicembre 2015)

10. https://www.asco.org/guidelines (Accesso 1 dicembre 2015)

11. http://iom.nationalacademies.org (Accesso 1 dicembre 2015)

12. http://www.snlg-iss.it (Accesso 1 dicembre 2015)

13. IOM (1990) Clinical practice guidelines: directions for a new program. National Academy Press, Washington DC

14. IOM (1992) Guidelines for clinical practice: from development to use. National Academy Press, Washington DC

15. Kung J, Miller RR, Mackowiak PA (2012) Failure of Clinical Practice Guidelines to meet Institute of Medicine Standards. Two more decades of little, if any, progress. Arch Intern Med $172: 1628-1633$

16. IOM (2008) Knowing what works in health care: a roadmap for the Nation. National Academy Press, Washington DC

17. Cabana MD, Rand CS, Powe NR et al (1999) Why don't physicians follow Clinical Practice Guidelines? A framework for improvement. JAMA 282:1458-1465

18. IOM (2009) Conflict of interest in medical research, education, and practice. National Academy Press, Washington DC

19. IOM (2011) Clinical Practice Guidelines we can trust. National Academy Press, Washington DC

20. Bossuyt PM, Reitsma JB, Bruns DE (2015) STARD 2015: an updated list of essential items for reporting diagnostic accuracy studies. BMJ 351:h5527

21. Aakre KM, Langlois MR, Watine J et al (2013) Critical review of laboratory investigations in clinical practice guidelines: proposals for the description of investigation. Clin Chem Lab Med 51:12171226

22. http://www.cap.org/web/home/resources/cap-guidelines?_afrLoop $=2295555404233631$ (Accesso 1 dicembre 2015) 
23. https://www.rcpath.org/resource-library-homepage/clinicaleffectiveness/clinical-guidelines.html (Accesso 1 dicembre 2015)

24. Wenham PR (2013) The annals as a disseminator of guidelines and promoter of best practice. Ann Clin Biochem 50:383-385

25. Hayward CP, Moffat KA, George TI et al (2015) Assembly and evaluation of an inventory of guidelines that are available to support clinical hematology laboratory practice. Int J Lab Hematol 37(Suppl 1):36-45

26. https://www.escmid.org/escmid_library/medical_guidelines/ escmid_guidelines/ (Accesso 1 dicembre 2015)

27. https://www.aacc.org/community/national-academy-of-clinicalbiochemistry/practice-guidelines (Accesso 1 dicembre 2015)

28. IOM (2011) Finding what works in health care-standards for systematic reviews. National Academy Press, Washington DC

29. NACB (2014) Standard operating procedures for preparing, publishing and revising National Academy of Clinical Biochemistry Laboratory Medicine Practice Guidelines. Including review and approval of external society/organization guidelines for endorsement and support by AACC/NACB. https://www.aacc.org/ / media/files/nacb/nacb_lmpg_sop_jan_2014.pdf?la=en (Accesso 1 dicembre 2015)

30. Don-Wauchope AC, Sievenpiper JL, Hill SA et al (2012) Applicability of the AGREE II instrument in evaluating the development process and quality of current National Academy of Clinical Biochemistry Guidelines. Clin Chem 58:1426-1437

31. Brouwers MC, Kho ME, Browman GP et al (2010) AGREE II: advancing guideline development, reporting and evaluation in health care. CMAJ 182:e839-e842
32. The AGREE Enterprise Website. Appraisal of guidelines for research \& evaluation (AGREE) instrument. http://www.agreetrust. org (Accesso 1 dicembre 2015)

33. Guyatt GH, Oxman AD, Vist GE et al (2008) GRADE: an emerging consensus on rating quality of evidence and strength of recommendations. BMJ 336:924-926

34. Schünemann HJ, Oxman AD, Brozek J et al (2008) Grading quality of evidence and strength of recommendations for diagnostic tests and strategies. BMJ 336:1106-1110

35. http://www.uspreventiveservicestaskforce.org/ (Accesso 1 dicembre 2015)

36. Misra S, Barth JH (2013) Guidelines are written, but are they followed? Ann Clin Biochem 50:400-402

37. Greco PJ, Eisenberg JM (1993) Changing physicians' practice. N Engl J Med 329:1271-1273

38. SIPMeL (2014) Statuto. http://www.sipmel.it/it/documento.php/ 106362 (Accesso 1 dicembre 2015)

39. SIPMeL (2012) Regolamento dei gruppi di studio. http://www. sipmel.it/it/documento.php/1813 (Accesso 1 dicembre 2015)

40. Shojania KG, Sampson M, Ansari MT et al (2007) How quickly do systematic reviews go out of date? A survival analysis. Ann Intern Med 147:224-233

41. Price CP, Christenson RH (2007) Evidence-based laboratory medicine: principles, practice and outcomes, 2nd edn. AACC Press, Washington DC

42. Cappelletti P (2005) La risposta della Medicina di Laboratorio al quesito clinico. RIMeL/IJLaM 1(Suppl):15-23 